\title{
ROTATIONAL MISMATCH OF SELF-ALIGNED TIBIAL COMPONENT IN POSTERIOR-STABILIZED (PS) TOTAL KNEE ARTHROPLASTY
}

\author{
Saradej Khuangsirikul, Montri Choowong, Songpol Trakulngernthai, Thanainit Chotanaphuti \\ Department of Orthopedics, Phramongkutklao College of Medicine, Bangkok, Thailand
}

Background: Rotational alignment of femoral and tibial prosthesis is one of the important factors for outcomes of total knee arthroplasty (TKA). Rotational malalignment may lead to patellar maltracking, anterior knee pain, femoro-tibial flexion instability and premature wear of the polyethylene inlay. Several studies have demonstrated higher revision rates and less favorable clinical results among patients with rotational malalignment. The transepicondylar axis is widely accepted as the best representation of the functional flexion-extension axis of the knee. On the other hand, no comparable agreement exists for tibial rotational alignment.

Objectives: The aim of this study was to determine the accurate rotational alignment of components by posterior cruciate ligament substituting TKA using the center-post self-align technique.

Methods: From January 2007 to May 2009, 54 patients (60 knees) underwent the cemented, posterior cruciate ligament substituting TKA using the center-post self-align technique of the tibial component and performed computer tomography postoperatively. The rotational angle between the femoral and tibial components and the rotational variance from the transepicondylar axis were measured.

Results: The rotational alignment of femoral components were $90 \%$ in the neutral group: $48.3 \%$ external rotate (mean $1.15^{\circ}$, range $0.1^{\circ}-4.9^{\circ}$ ), $48.3 \%$ internal rotate (mean $1.53^{\circ}$, range $0.2^{\circ}-3.8^{\circ}$ ) and $3.4 \%$ were in neutral alignment. The rotation alignment of tibial components were $71.7 \%$ in the neutral group: $41.2 \%$ external rotate (mean $2.03^{\circ}$, range $0.2^{\circ}-6.7^{\circ}$ ), $56.7 \%$ internal rotate (mean $2.59^{\circ}$, range $0.3^{\circ}-6.7^{\circ}$ ) and $1.67 \%$ had neutral alignment. We found no rotational mismatch between femoral and tibial components in this study. All 60 knees had good patellar tracking by no thumb test technique without lateral released procedure.

Conclusion: Femoral component rotations were mostly in the safe zone. Using the center-post self-align technique in posterior cruciate ligament substituting TKA, tibial component rotation was much more varied than the femur. However, all tibial component rotations were in between medial most and medial $1 / 3$ of the tibial tuberble.

Keywords : Rotational, TKA, mismatch, self align, tibial component

J Southeast Asian Med Res 2017; 1: 48-52.

http://www.jseamed.org

\section{Correspondence to:}

Chotanaphuti T, Department of Orthopedics, Phramongkutklao College of Medicine, Bangkok, Thailand

E-mail : tanainit@hotmail.com 


\section{Introduction}

The outcomes of total knee arthroplasty (TKA) depend on multiple factors. In addition to the patient-related factors, surgical factors such as mechanical axis restoration, rotational alignment and soft tissue balancing also affect the good long-term results. Transepicondylar axis, widely accepted as the best representation of the functional flexion-extension axis of the knee, has been used as a reference for femoral rotational alignment. ${ }^{(1-5)}$ However, a standard reference for tibial rotational alignment remains controversial. Rotational malalignment may lead to patellar maltracking, anterior knee pain, femoro-tibial flexion instability and premature wear of the polyethylene inlay. ${ }^{(6-10)}$ Several studies have reported higher revision rates and less favorable clinical results among TKA patients with rotational malalignment. ${ }^{(8,11,12)}$ Plastic deformation and gross damage to the tibial post are the results of anterior or posterior impingement against the femoral component. ${ }^{(13-15)}$

Akagi et al. ${ }^{(16)}$ measured the angles between a line perpendicular to the transepicondylar axis and different landmarks on healthy subjects. They reported that an axis from the medial border of the ligamentum patellae to the posterior cruciate ligament has the lowest variability among volunteers. However, because this study was conducted on nonosteoarthritic knees, its application to osteoarthritic knees is questionable.

Other different landmarks have been used for tibial rotational alignment. Currently, two techniques are widely used to determine tibial rotational alignment among TKR patients. ${ }^{(17)}$ The first is anatomical landmarks such as tibial tuberosity, posterior condylar line of the tibia and malleolar axis of ankle and the second is the range of movement (ROM) technique. The ROM technique, in which the knee is moved through a full range of flexion and extension, allows the tibial trial to orientate itself in the best position relative to the femoral component. With this technique, the anterior tibial cortex is marked and the tibial component is then implanted to match this mark. ${ }^{(18)}$ This method is based on the hypothesis that the rotational mismatch between femoral and tibial components should be zero degree when both components are ideally implanted using the range of movement (ROM) technique or center-post self-align technique.

\section{Methods}

This study was a prospective study and approved by the Ethics Committee of Phramongkutklao Hospital and Phramongkutklao College of Medicine. Informed consent was obtained from all subjects.

Patients undergoing TKA in Phramongkutklao Hospital from January 2007 to May 2009 were eligible for the study. Inclusion criteria included 1) patients with primary or secondary osteoarthritis of the knee, 2) age more than 55 years, 3) no previous partial or total knee arthroplasty, 4) deformity between $15^{\circ}$ of varus and $5^{\circ}$ of valgus and 5) no severe instability. Because we used postoperative computerized tomography (CT) to evaluate the outcome, patients who refused postoperative $\mathrm{CT}$ were excluded from the study.

\section{Surgical technique}

All procedures were performed by a single senior surgeon. Posterior cruciate ligament substituting TKA (PFC Sigma, Depuy, Warsaw, Indiana), fixed bearing tibial component and an all-polyethylene patellar component were implanted in all patients, with surgical self-aligned technique for tibial rotation. A standard midline incision and medial parapatellar approach were performed. The femoral bone cut was created by intramedullary guide and anterior sizing reference with 3-degree external rotation from posterior condylar axis. The tibial bone cut was created by extramedullary guide. The tibial rotational alignment was performed by center-post self-align technique after the femoral component trial was placed. The knee was passively flexed and extended five times, allowing the unsecured tibial trial to seek its own rotation which occurred between the undersurface of the unsecured tibial trial and the cut surface of the proximal tibia. The rotational orientation of the tibial trial determined by the ROM technique was marked on the anterior tibial cortex by electrocautery and patellar tracking was evaluated intra-operatively. Fig. 1

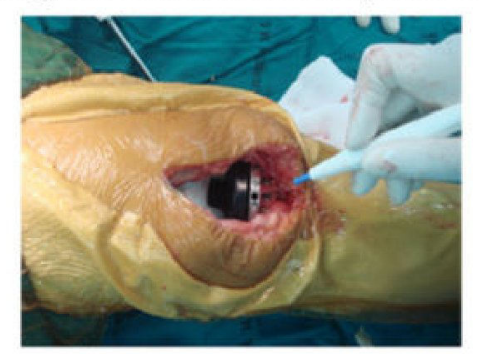

Fig. 1 The mark of tibial component with center-post self-align technique was made by electrocauterization. 


\section{Radiographic and CT evaluation}

All patients performed a radiographic and CT assessment at five to seven days after surgery to evaluate the leg axis and alignment of the components. The patellar tilt and the displacement of the patella were measured on a Laurin view radiograph. The patellar tracking was defined as neutral when the tilt was within $\pm 10^{\circ}$ and displacement less than $5 \mathrm{~mm} .{ }^{(19)}$ The CT digital images were evaluated in fully extended position of the knee in the supine position using the software ID, PACS Release 3.6 (Image Devices, Idstein, Germany). The rotational alignment of the femoral component was defined as a line through the edge of both posterior condylars of femoral prostheses. The rotational alignment of the tibial component was defined as a line along the posterior border of the tibial tray. The rotational alignment of the femoral and tibial components were then superimposed and the mismatch between both was measured. The rotational alignment of the patellar component was defined as a line along the surface bone cutting beneath the polyethylene component. All imagination lines were compared with the epicondylar axis line of the femur.

Fig. 2
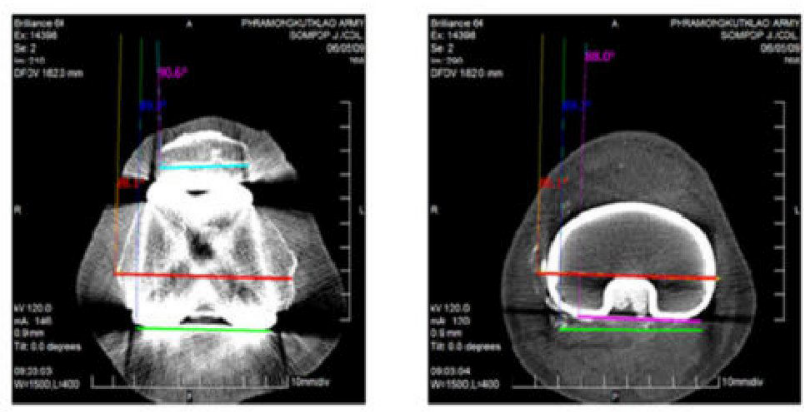

Fig. 2 The computerized tomography images were performed in the fully extended knee, in the supine position and component alignment was measured using digital software.

The femoro-tibial component rotational mismatch was defined as the difference between femoral and tibial components on CT images. True femoro-tibial rotational mismatch was defined as the difference between an ideal position of the femoral component (transepicondylar line) and the tibial component (center-post self-align technique). Our study used 10-degree mismatch as the reference point because the biomechanical study demonstrated an increased tibial cortical strain in the TKA that had femoro-tibial rotational mismatch more than 10 degrees. ${ }^{(20)}$

\section{Results}

Sixty TKAs in 54 patients were included in the study; $11.1 \%$ ( 6 patients) were male and $88.9 \%$ (48 patients) were female. Mean age was 69 years (range, 63 to 79 years).

Rotational alignments of the femoral component comprised $48.3 \%$ (29 knees) external rotation from transepicondylar axis (mean $1.15^{\circ}$, range $0.1^{\circ}-4.9^{\circ}$ ), $48.3 \%$ (29 knees) internal rotation (mean $1.53^{\circ}$, range $0.2^{\circ}-3.8^{\circ}$ ) and $3.4 \%$ ( 2 knees) in neutral alignment.

Rotational alignment of the tibial component comprised $41.2 \%$ ( 25 knees) external rotation from transepicondylar axis (mean $2.03^{\circ}$, range $0.2^{\circ}-6.7^{\circ}$ ), $56.7 \%$ (34 knees) internal rotation (mean $2.59^{\circ}$, range $0.3^{\circ}-6.7^{\circ}$ ) and only 1 knee $(1.67 \%)$ was in neutral alignment. All tibial component rotations were between the medial most and medial $1 / 3$ of the tibial tuberble.

Mean rotational mismatch between tibial and femoral components was $2^{\circ}\left(\right.$ range $\left.0.1^{\circ}-5.8^{\circ}\right)$. No patients had femoro-tibial mismatch over $10^{\circ}$ (range $0.1^{\circ}-5.8^{\circ}$ )

The patellar rotational axis comprised $63.33 \%$ (39 knees) external rotation from transepicondylar axis (mean $5.85^{\circ}$, range $\left.0.6^{\circ}-18.8^{\circ}\right)$ and $36.67 \%$ (21 knees) internal rotation (mean $4.12^{\circ}$, range $0.2^{\circ}-17.2^{\circ}$ ). Fig. 3-5

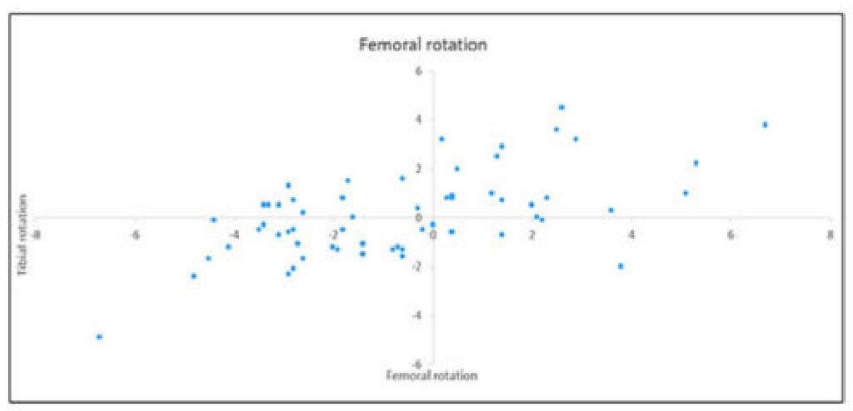

Fig. 3 Alignment characteristics from computer tomography compared between femoral and tibial components

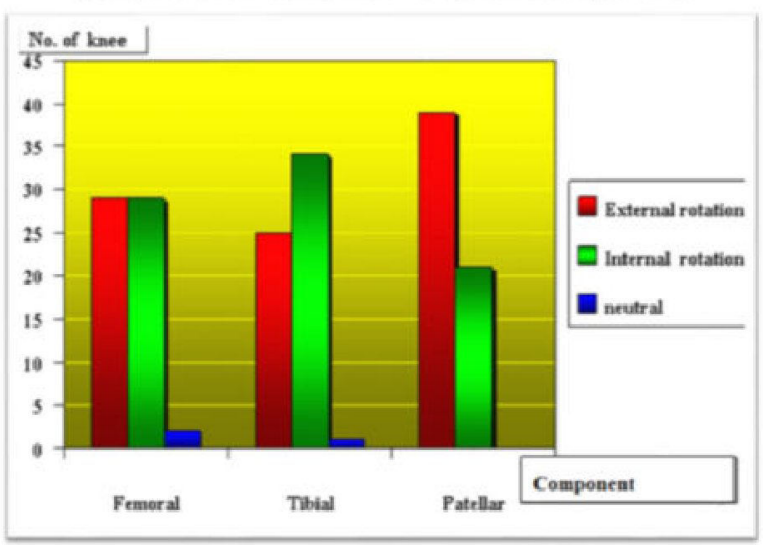

Fig. 4 Rotational alignment characteristics from computer tomography following TKA 


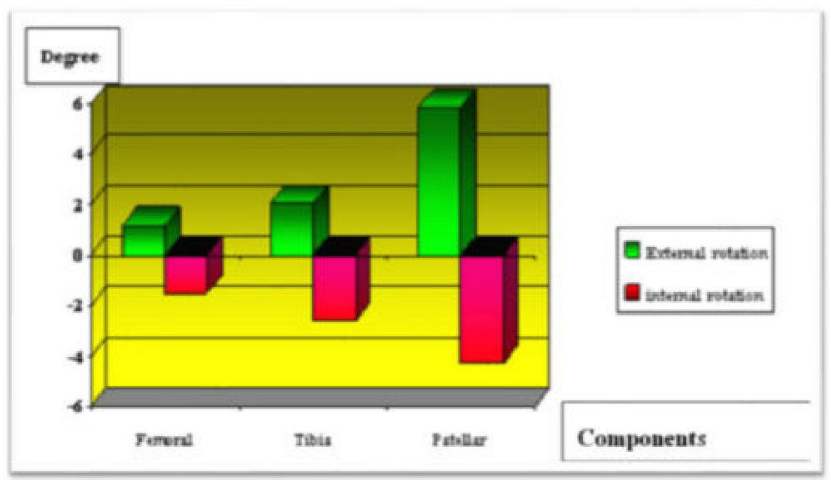

Fig. 5 Mean rotational component from computer tomography following TKA

All 60 TKAs had good patellar tracking by the no thumb test technique without lateral released procedure. Radiographic evaluation of patellar orientation with Laurin film found $90 \%$ (54 knees) within normal range $\left( \pm 10^{\circ}\right.$ patella tilt and less than $5 \mathrm{~mm}$ displacement).

Mean distance between tibial tuberosity and trochea groove (TT-TG) was $5.29 \mathrm{~mm}$ (range, $1.0-14.3 \mathrm{~mm}$ ).

\section{Discussion}

The transepicondylar axis has been widely used as a reference for femoral rotational alignment. ${ }^{(1-5)}$ On the other hand, no comparable agreement exists for tibial rotational alignment.

Several studies aligned the tibial component using the landmark of the medial $1 / 3$ of the tibial tuberosity resulting in occasionally excessive external rotation in some cases. ${ }^{(21-23)}$ Eckhoff et al. ${ }^{(23)}$ documented the amount of malrotation between the femoral and tibial components with a contemporary alignment technique. They reported the average external rotation of the tibial component relative to the femoral component associated with the reference of tibial tuberosity was $19^{\circ}$ and those rotational mismatches might account for posteromedial polyethylene wear. Uehara et al. ${ }^{(21)}$ demonstrated a tendency to align the tibial component in external rotation relative to the femoral component using computed tomography. He reported rotational mismatch in nearly $50 \%$ of the subjects between the axis of medial $1 / 3$ of the tibial tuberosity and the transepicondylar axis for the femur. Another possible cause of the difference was medial torsion of the tibia. Nagamine et al. ${ }^{(24)}$ demonstrated that the foot could be severely rotated internally when the medial $1 / 3$ of the tibial tuberosity was used as a guide for rotational alignment among patients with severe medial torsion of the tibia. In patients from East Asian countries, the medial torsion of the tibia should be taken into account, because it can aggravate rotational mismatch. Tang et al ${ }^{(25)}$ showed a tendency for the tibial component to be externally rotated when the medial one third of the tibial tuberosity was defined as a rotational landmark. This finding was particularly prominent in Chinese osteoarthritic knees with varus or valgus deformities. The anteroposterior axis of the tibia intersected with the patellar ligament near its medial $10 \%$ in healthy Chinese knees, whereas it intersected the medial $20 \%$ in varus knees and the medial $30 \%$ in valgus knees.

According to the posterior cruciate ligament substituting TKA (PFC Sigma, Depuy, Warsaw, Indiana), the tibial post was designed to engage the femoral cam at approximately $42^{\circ}$ of knee flexion and through the endpoint of flexion. Furthermore, the post could accommodate up to $8^{\circ}$ of internal or external rotation between the femur and tibia from the neutral axis before impinging against the femoral box. The Sigma posterior-stabilized prosthesis generated little torque through $5^{\circ}$ internal and external rotation. An increase in torque then occurred because of box-post impingement, generating peak torques of 17 to $18 \mathrm{~N}-\mathrm{m}$ at $12^{\circ}$ to $14^{\circ}$ rotation. Small changes in relative tibio-femoral component rotation can more than double the generated torque. Axial rotation of the knee in vivo can generate substantial torque. Relative tibio-femoral rotational position is an important factor influencing component function and fixation. ${ }^{(26)}$

\section{Conclusion}

We found no femoro-tibial rotational mismatch among patients undergoing posterior-stabilized (PS) total knee arthroplasty with center-post self-align technique. All tibial component rotations were between the medial most and medial $1 / 3$ of the tibial tuberble. This technique can reduce femoro-tibial rotational mismatch with good patella tracking for posterior cruciate ligament substituted TKA. The long term results and functional outcomes should be further investigated.

\section{References}

1. Asano T, Akagi M, Nakamura T. The functional flexionextension axis of the knee corresponds to the surgical epicondylar axis: in vivo analysis using a biplanar 
image-matching technique. J Arthroplasty 2005; 20: 1060-7.

2. Churchill DL, Incavo SJ, Johnson CC, Beynnon BD. The transepicondylar axis approximates the optimal flexion axis of the knee. Clin Orthop Relat Res 1998; 356: 111-8.

3. Miller MC, Berger RA, Petrella AJ, Karmas A, Rubash HE. Optimizing femoral component rotation in total knee arthroplasty. Clin Orthop 2001; 392:38-45.

4. Akagi M, Mori S, Nishimura S, Nishimura A, Asano T, Hamanishi C. Variability of extraarticular tibial rotation references for total knee arthroplasty. Clin Orthop Relat Res 2005; 436: 172-6.

5. Olcott CW, Scott RD. The Ranawat Award. Femoral component rotation during total knee arthroplasty. Clin Orthop Relat Res 1999; 367: 39-42.

6. Barrack RL, Schrader T, Bertot AJ, Wolfe MW, Myers L. Component rotation and anterior knee pain after total knee arthroplasty. Clin Orthop Relat Res 2001; 392: 46-55.

7. Berger RA, Crossett LS, Jacobs JJ, Rubash HE. Malrotation causing patellofemoral complications after total knee arthroplasty. Clin Orthop 1998; 356: 144-.53.

8. Hofmann S, Romero J, Roth-Schiffl E, Albrecht T. Rotational malalignment of the components may cause chronic pain or early failure in total knee arthroplasty. Orthopade 2003; 32: 469-76.

9. Insall JN, Scuderi GR, Komistek RD, Math K, Dennis DA, Anderson DT. Correlation between condylar lift-off and femoral component alignment. Clin Orthop Relat Res 2002; 403: 143-.52.

10. Wasielewski RC, Galante JO, Leighty RM, Natarajan RN, Rosenberg AG. Wear patterns on retrieved polyethylene tibial inserts and their relationship to technical considerations during total knee arthroplasty. Clin Orthop Relat Res 1994; 299: 31-.43.

11. Romero J, Stahelin T, Binkert C, Pfirrmann C, Hodler J, Kessler $\mathrm{O}$. The clinical consequences of flexion gap asymmetry in total knee arthroplasty. J Arthroplasty 2007; 22 : 235-40.

12. Incavo SJ, Wild JJ, Coughlin KM, Beynnon BD. Early Revision for Component Malrotation in Total Knee Arthroplasty. Clin Orthop Relat Res 2007; 458: 131-6.

13. Callaghan JJ, O'Rourke MR, Goetz DD, Schmalzried TP, Campbell PA, Johnston RC. Tibial post impingement in posterior-stabilized total knee arthroplasty. Clin Orthop Relat Res 2002; 404: 83-8.

14. Banks SA, Harman MK, Hodge WA. Mechanism of anterior impingement damage in total knee arthroplasty. J Bone Joint Surg Am 2002; 84-A(Suppl 2): 37-42.
15. Puloski SK, McCalden RW, MacDonald SJ, Rorabeck CH, Bourne RB. Tibial post wear in posterior stabilized total knee arthroplasty. An unrecognized source of polyethylene debris. J Bone Joint Surg Am 2001; 83-A: 390-7

16. Olcott CW, Scott RD. A comparison of 4 intraoperative methods to determine femoral component rotation during total knee arthroplasty. J Arthroplasty 2000; 15 : 22-6.

17. Chowdhury EA, Porter ML, et al: How is the tibial tray aligned to the femoral prosthesis in a total knee arthroplasty?: a survey of opinion from BASK? Knee 2005; 12: 79-80.

18. Churchill DL, Incavo SJ, Johnson CC, Beynnon BD. The transepicondylar axis approximates the optimal flexion axis of the knee. Clin Orthop Relat Res 1998; 356: 111-8.

19. Heesterbeek PJC, Beumers MPC, Jacobs WCH, Havinga $\mathrm{ME}$, Wymenga $\mathrm{AB}$. A comparison of reproducibility of measurement techniques for patellar position on axial radiograph after total knee arthroplasty. Knee 2007; 14; 411-6.

20. Kessler O, Lacatusu E, Sommers MB, Mayr E, Bottlang M. Malrotation in total knee arthroplasty: Effect on tibial cortex strain captured by laser-based strain acquisition. Clin Biomech (Bristol, Avon) 2006; 21: 603-9,

21. Uehara K, Kadoya Y, Kobayashi A, Ohashi H, Yamano $\mathrm{Y}$. Bone anatomy and rotational alignment in total knee arthroplasty. Clin Orthop Relat Res 2002; 402: 196-201.

22. Chowdhury EA, Porter ML. How is the tibial tray aligned to the femoral prosthesis in a total knee arthroplasty?: a survey of opinion from BASK? Knee 2005; 12: 79-80.

23. Eckhoff DG, Metzger RG, Vandewalle MV. Malrotation associated with implant alignment technique in total knee arthroplasty. Clin Orthop 1995; 321: 28-31.

24. Nagamine R, Miyaanishi K, Miura H, Urabe K, Matsuda S, Iwamoto Y. Medial torsion of the tibia in Japanese patients with osteoarthritis of the knee. Clin Orthop 2003; 408: 218-24.

25. Sun T, Lu H, Hong N, Wu J, Feng C. Bony landmarks and rotational alignment in total knee arthroplasty for Chinese osteoarthritic knees with varus or valgus deformities. J Arthroplasty 2009; 24: 427-31.

26. 26.Klein R, Serpe L, Kester MA, Edidin A, Fishkin, Z, Mahoney, O. et al. Rotational constraint in posteriorstabilized total knee prostheses. Clin Orthop Relat Res 2003; 410: 82-9. 